Reviews

\title{
Recent Nanoarchitectures in Metal Nanoparticle-modified Electrodes for Electroanalysis
}

\author{
Munetaka OYAMA \\ Department of Material Chemistry, Graduate School of Engineering, Kyoto University, \\ Nishikyo, Kyoto 615-8520, Japan
}

\begin{abstract}
Increasing attention has been devoted to the use of metal nanoparticles (NPs) for electroanalysis. To make the best use of the electrocatalytic and electron-conducting characteristics of metal NPs, various nanoarchitectures have been developed for modifying metal NPs on electrode surfaces. In this review, at first recent nanoarchitectures with metal NPs for modifying electrodes are summarized together with the results of electrochemical analysis. Then, the progress of a seed-mediated growth method that we developed for modifying electrode surfaces is shown as an example that the nanoarchitectures of metal NPs are possible without using organic linker molecules. This approach should be effective for further functional modifications of the surfaces of metal NPs as well as the electrochemical analysis with lower charge-transfer resistance.
\end{abstract}

(Received October 30, 2009; Accepted December 11, 2009; Published January 10, 2010)

1 Introduction

2 Electrochemical Deposition of Metal NPs

3 Modification of Chemically Prepared Metal NPs-Typical Methods and Problems-

4 Linker Molecules to Attach Metal NPs

5 Self-assembly on a Three-dimensional Sol-gel Silica Network

6 Polymer Materials for Modifying Metal NPs

7 Integrated Nanoarchitectures with Metal

NPs for Modifying Electrodes

8 Seed-mediated Growth Method for Modifying Electrode Surfaces

8.1 Development of a seed-mediated growth method for surface modification
8.2 Electrochemical applications of AuNPattached ITO

8.3 Improved methods for the attachment and growth of AuNPs on ITO

8.4 Attachment and growth of Au nanoplates on ITO

8.5 Attachment and growth of AuNPs on GC and $\mathrm{TiO}_{2}$

8.6 Attachment and growth of other metal NPs on ITO

8.7 Electrochemical measurements of biomolecules using AuNP/ITO electrodes

9 Concluding Remarks 9

10 References

\section{Introduction}

Metal nanoparticles (NPs) have been attracting huge attention during this decade because of their unique optical, electronic, magnetic and catalytic properties. In the field of electrochemistry, in particular electroanalysis, metal NPs have been actively utilized as functional nanomaterials. There are some excellent review articles up to now concerning the use of metal NPs in electroanalysis. $^{1-4}$ While some parts of earlier reviews ${ }^{1,2}$ dealt with the utilization of NPs as conducting base nanomaterials for further modifications (such as for immunosensing and DNA labeling), the modification of metal NPs on conducting materials, i.e., electrode surfaces, is apparently effective in electrochemical analysis. Therefore, the applications of metal NPs to modified electrodes have been a main focus in recent reviews. ${ }^{3,4}$

E-mail: m.oyama@kx8.ecs.kyoto-u.ac.jp
In a review by Welch and Compton in $2006,{ }^{3}$ it is mentioned that there are four main advantages to using NP-modified electrodes with regard to electroanalysis: high effective surface area, mass transport, catalysis and control over the local micro-environment, by referring a previous review $^{2}$ in the introduction. Also, the progress of metal NP-modified electrodes up to 2005 has been well summarized according to the sort of metals, i.e., gold, silver, platinum, nickel, copper, iron and other metals. For gold, silver and platinum, the descriptions have been separated into two parts by the preparation methods, i.e., "colloidal production" and "deposition using electrochemistry". A review by Lawrence and Liang in $2008^{4}$ also focused on metal-NP modified electrodes. In their review, ${ }^{4}$ at first, one table is dedicated to a comparison of the applications of metal NPs in electroanalysis, while explicitly listing the nanoparticles, analyte, methodology, dynamic range and detection limit for 98 results. Then, the electroanalytical applications have been reviewed concerning NPs of gold, platinum, silver, palladium, 
copper, nickel, iron and others, in this order.

Under the circumstances that the results of electroanalysis with metal NP-modified electrodes have been well reviewed, depending on the sort of metal, in the present article, at first, we focus on recent developments of nanoarchitectures, or nanostructuring, of the electrode interfaces with metal NPs. After featuring some recent successful examples of nanoarchitectures in metal NP-modified electrodes for electrochemical analysis, we summarize the progress of a seed-mediated growth method that we developed for modifying electrode surfaces as an interesting example that nanoarchitectures of metal NPs are possible without using organic linker molecules.

\section{Electrochemical Deposition of Metal NPs}

Electrochemical deposition would be a powerful method to prepare metal-NP modified electrodes. Because the nucleation and growth of metal NPs occur directly on a conducting surface during electrolysis, the attachment of metal NPs should be strong enough, and the conducting communication between the base conductors and metal NPs should be quite smooth. However, the electrolysis reaction does not tend to proceed uniformly, so careful controls of the electrolysis conditions would be necessary for preparing metal-NPs having size uniformity and controlled densities.

Finot et al. reported on the characterization of interfacial properties of gold nanocrystals deposited electrochemically on glassy carbon (GC) substrates, ${ }^{5}$ together with well-summarized previous trials for electrodepositing $\mathrm{Au}$ on carbon electrodes. Their results showed that the overall surface area of gold as well as the particle size, density, and surface texture can be controlled by varying the deposition conditions. ${ }^{5}$ El-Deab et al. reported on the control of the loading of gold NPs (AuNPs) on GC for the electrochemical reduction of oxygen. ${ }^{6}$ By changing the concentration of $\mathrm{AuCl}_{4}{ }^{-}$and the deposition time, the changes in the Au loading was clearly shown by SEM images, which would be a beautiful example of the tuning of the interfacial nanostructure of AuNPs. Furthermore, the same group reported interesting crystallographic orientation control of AuNPs on GC electrodes in the presence of two different additives (cystein and iodide ions) (Fig. 1). ${ }^{7}$ The direct electron transfer of copper-zinc superoxide dismutase was realized for the first time using thus-prepared crystallographically oriented AuNP-deposited GC electrodes. ${ }^{8}$

AuNP-electrodeposited GC electrodes prepared by a potential step method have been utilized for an anodic stripping voltammeric analysis of $\mathrm{As}(\mathrm{III})$ in $1 \mathrm{M} \mathrm{HCl}$ by Dai et al. ${ }^{9}$ In addition, a flow analysis electrochemical system for the detection of As(III) has been developed using an AuNP-modified GC electrode prepared with a similar electrodeposition by Majid et al. ${ }^{10}$ Carralero Sanz et al. reported a biosensor based on a cross-linking immobilization of tyrosinase with glutaraldehyde onto a GC electrode modified with electrodeposited AuNPs, which was applied to the amperometric analysis of polyphenols in wines. ${ }^{11}$

In addition to GC substrates, recent advances have been devoted to the electrochemical deposition of AuNPs on other substrates. Dai and Compton reported the preparation of AuNP-modified indium tin oxide (ITO) film coated glass electrodes for the first time through direct electrochemical deposition from $0.5 \mathrm{M} \mathrm{H}_{2} \mathrm{SO}_{4}$ containing $0.1 \mathrm{mM} \mathrm{HAuCl}{ }_{4}{ }^{12}$ The resulting surfaces were characterized with AFM and utilized for the detection of As(III).
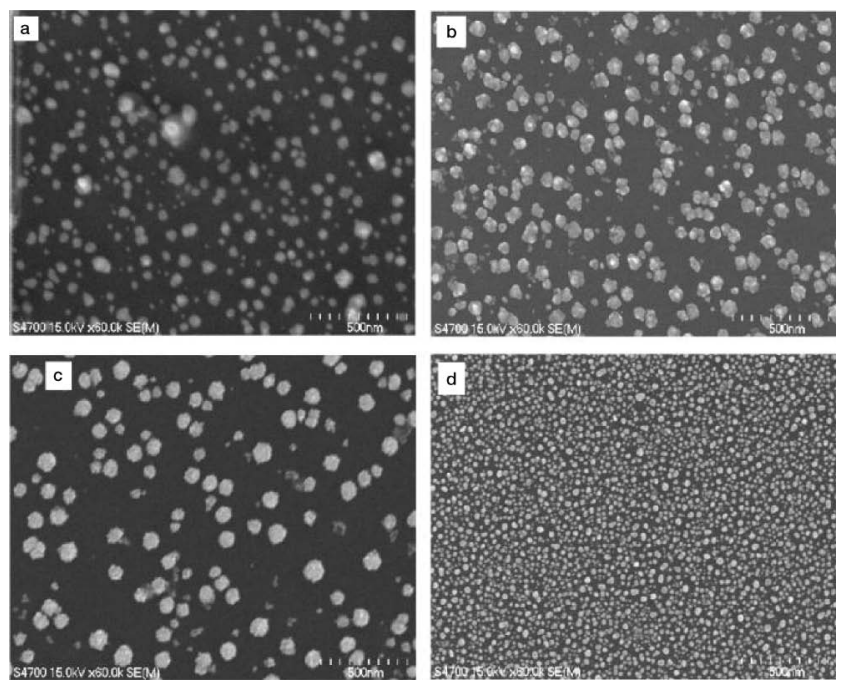

Fig. 1 SEM images for the AuNPs electrodeposited on GC electrodes from $0.5 \mathrm{M} \mathrm{H}_{2} \mathrm{SO}_{4}$ containing $1.0 \mathrm{mM} \mathrm{AuCl}_{4}^{-}$in (a) the absence of cysteine and $\mathrm{I}^{-}$, (b) the presence of $10 \mu \mathrm{M}$ cysteine, (c) $100 \mu \mathrm{M}$ cysteine, and (d) $100 \mu \mathrm{M} \mathrm{I}^{-}$ions. The potential was stepped from 1.1 to $0 \mathrm{~V}$ for $60 \mathrm{~s}$. Reproduced from Ref. 7, copyright 2005, with permission from the Electrochemical Society.

While $\mathrm{AuCl}_{4}{ }^{-}$was normally used for electrodeposition, Wang et al. reported on the direct electrodeposition of AuNPs onto ITO using a $\mathrm{KAu}(\mathrm{CN})_{2}$ phosphate buffer solution. The electrochemical deposition was performed by a cyclic voltammetric mode at $50 \pm 2{ }^{\circ} \mathrm{C}$. As a result, AuNPs of $20-35 \mathrm{~nm}$ were directly attached on the ITO surfaces with a quasi-spherical shape and a quasi-symmetric distribution. Thus-prepared AuNP-deposited ITO coated glasses were used for the immobilization of superoxide dismutase, which realized direct electron transfer, and for the detection of a superoxide anion. ${ }^{13}$ Using the same electrodes, the same group reported on the electrocatalytic oxidation of glucose ${ }^{14}$ a horseradish peroxidase-based hydrogen peroxide sensor, $^{15}$ and the amperometric detection of morphine. ${ }^{16}$

As an example of a different substrate and Au precursor, Rassaei et al. reported on the electroaggregation of commercially available 5-nm AuNPs on a boron-doped diamond electrode surface. ${ }^{17}$ The electroaggregation process formed a highly reactive mesoporous $\mathrm{Au}$ film, which was applied to an electrochemical analysis of As(III).

In addition to AuNPs, silver NPs (AgNPs) was electrodeposited on a GC electrode, and electrocatalytic action for the reduction of benzyl chloride was reported by Isse et al. ${ }^{18}$ Palladium NPs (PdNPs) could be also prepared on GC and ITO electrodes by simple electrochemical deposition process; the effectiveness in the detection of catecholamines in the presence of ascorbic acid was shown by differential pulse voltammerty by Thiagarajan et al. ${ }^{19}$ With electrochemically deposited platinum NPs (PtNPs) modified GC electrodes, Aoun and Taniguchi reported on the direct electrocatalytic oxidation of glucose in an alkaline solution. ${ }^{20}$

As a new substrate, Safavi et al. reported the formation of a PdNP-array by electrodeposition on a carbon ionic liquid electrode, which was prepared using graphite powder and octylpyridinum hexafluorophosphate with a ratio of 50/50 (w/w). ${ }^{21,22}$ The thus-fabricated electrode was applied to $\mathrm{O}_{2}$ reduction, $\mathrm{H}_{2} \mathrm{O}_{2}$ oxidation, ${ }^{21}$ and the oxidation of formaldehyde. ${ }^{22}$ 
Thiagarajan et al. reported on the absorption of $\mathrm{TiO}_{2}$ on GC and ITO electrodes, followed by the electrochemical deposition of nano $\mathrm{Au}$ and $\mathrm{KI}$ film for the electrocatalytic oxidation of $\mathrm{H}_{2} \mathrm{O}_{2} .{ }^{23}$

\section{Modification of Chemically Prepared Metal NPs-Typical Methods and Problems-}

The chemical preparation of metal colloid is another representative approach to prepare metal NP-modified electrodes. By chemically synthesizing metal NPs in solutions before modification, metal NPs having a well-controlled shape and size can be modified, or attached onto the electrode surfaces compared with the electrochemical deposition method. However, some functional bindings of metal NPs are necessary for the attachment or fixation on the electrode surfaces, though metal NPs might be modified on the electrode just by dropping a solution containing metal NPs in some cases. ${ }^{24-26}$ Thus, many trials have been devoted to the interfacial nanoarchitectures of metal NPs to prepare metal NP-modified electrodes up to now.

The use of bridging reagents, or linker molecules, would be a well-known approach. For example, when we would like to attach AuNPs onto the surfaces of glass, ITO or $\mathrm{SiO}_{2}$, 3-mercaptopropyl-trimethoxysilane (MPTMS), 3-aminopropyltrimethoxysilane (APTMS) or related compounds can be utilized due to the bonding ability of the silanol group and the affinity of the $-\mathrm{SH}$ or $-\mathrm{NH}_{2}$ group toward AuNPs. ${ }^{27-35}$ With the MPTMS linker, the fabrication of $\mathrm{Au}$ nanoelectrode ensembles, ${ }^{30}$ and fine tuning ${ }^{31}$ and the manipulation of the AuNPs' growth on glass ${ }^{32}$ have been reported. On the other hand, AuNPs attached with the APTMS linker have been used for further Au nanostructuring on $\mathrm{SiO}_{2}{ }^{33}$ or glass, ${ }^{34}$ and the fabrication of a colloidal Au-modified ITO electrode. ${ }^{35}$

However, when linker molecules are used, it is expected that the organic moiety would interfere with the electron-transfer reactions. For example, the alkyl-chain length of the linker molecules should affect the electron-transfer reactions. Although the attachment of metal NPs at the outer terminal side of linker would recover the electrochemical communications, compared with the modification of only linker molecules, some drawbacks of organic component should be kept in mind to evaluate the performances of the modified electrodes under the view of electron transfer.

In addition to linker molecules, capping reagents should also affect the electron-transfer reactions and electrocatalytic functions of metal NPs. In the development of the synthesis of AuNPs, the Brust-Schiffrin method ${ }^{36}$ would really be a breakthrough to prepare a large amount of very small AuNPs with uniform-size utilizing thiols. Because the synthesized AuNPs are easily separated, the thiol-capped AuNPs might be assumed to be a component of the AuNP-modified electrode. However, thiol capping is too strong to obtain good electrochemical results, though the electrochemistry of thus-formed monolayer-protected Au clusters was extensively studied. $^{37}$ To evaluate the performance of metal NP-modified electrodes for electrolytic analysis, we should also note the selection of capping reagents.

In spite of the drawbacks of thiol capping, successful electrochemical results have been obtained after removal of the capping layer. Tominaga et al. reported detailed electrocatalytic activity of $\mathrm{Au},{ }^{38,39} \mathrm{Au}-\mathrm{Pt}$ alloy, ${ }^{38}$ and bimetallic $\mathrm{Au}-\mathrm{Ag} \mathrm{NPs}^{40}$ modified carbon electrodes for the oxidation of glucose after heat removal of the capped decanthiol at $300^{\circ} \mathrm{C}$. Yamamoto et al. reported the characteristics of AuNP film electrodes prepared on polystyrene, in which it was necessary to remove thiols by a successive voltammetric treatment in $0.1 \mathrm{M} \mathrm{H}_{2} \mathrm{SO}_{4}{ }^{41}$

As an example of removing other capping reagents, Chen et al. reported that the treatment in an ultraviolet ozone chamber was performed to remove oleylamine and oleic acid for using Fe-Pt alloy NPs on Au electrodes for electro-oxidation of formic

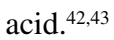

\section{Linker Molecules to Attach Metal NPs}

As mentioned above, MPTMS and APTMS would be typical linker molecules between AuNPs and glass, ITO or $\mathrm{SiO}_{2}$. In contrast, for the attachment of AuNPs on GC surfaces, benzothiol was bonded to GC via electro-reductive attachment using 4-mercaptobenzenediazonium tetrafluoroborate. ${ }^{44}$ Also, 4-aminobenzoic acid was covalently bonded to GC surfaces via electro-oxidation, and then 4-aminothiophenol was reacted to form peptide bonding while keeping the thiol terminals. ${ }^{45,46}$ Thus-fabricated AuNPs attached GC electrodes were applied to the electrochemistry of hemoglobin, ${ }^{45}$ ascorbic acid and dopamine. ${ }^{46} \mathrm{Hu}$ et al. reported that cysteamine could also be utilized in attaching AuNPs on GC via a cyclic voltammetric treatment to form covalent GC-N bonding, and that the AuNP-modified GC electrode could be used for a selective determination of dopamine in the presence of a high concentration of ascorbic acid. ${ }^{47}$

As an example to attach AuNPs onto a polycrystalline $\mathrm{Au}$ electrode, cystamine was used as a linker molecule. By forming an amine-terminated self-assembled monolayer, AuNPs were successfully immobilized on the amine-terminals, and a selective determination of dopamine could be performed. ${ }^{48}$ In contrast, L-cysteine, another molecule having an amino- and -SH terminal, was utilized to bridge a GC electrode and AuNPs, ${ }^{49}$ and an $\mathrm{Au}$ electrode and AgNPs. ${ }^{50}$

Regarding the attachment of AuNPs on Au electrodes, Sivanesan et al. reported a method to use 1,6-hexanedithiol for bridging, ${ }^{51}$ which was effective for the electroanalysis of ascorbic acid. The same group, Kannan and John, also reported the use of 2,5-dimercapto-1,3,4-thiadiazole to immobilize enlarged AuNPs for the electroanalysis of uric and ascorbic acids. ${ }^{52}$

In cases of $\mathrm{Au}$ base electrodes, mixed self-assembled monolayer systems have been utilized. Wang et al. reported a method using 4-aminothiophenol, followed by functionalization to the $4^{\prime}$-mercapto- $N$-phenylquinone diimine. ${ }^{53}$ In their trial, 1,4-benzenedimethanethiol was further modified to form a more compact and ordered platform to fabricate an AuNP-modified electrode. The same group reported an approach to use a mixed self-assembled monolayer of dithiothreitol and dodecanethiol for the same proposes, and applied it to the voltammetric sensing of epinephrine. ${ }^{54}$ El-Deab et al. reported on the fabrication of phase-separated multicomponent self-assembly monolayers at AuNP electrodeposited GC electrodes; the ternary self-assembly monolayer was composed of 1-dodecanethiol, 11-aminoundecanethiol, and 3-mercaptopropionic acid. ${ }^{55}$

Because several linker molecules can be used for modifying ITO electrodes, Ballarin et al. reported molecular effects for self-assembling AuNPs using MPTMS and 3-aminopropyltriethoxysilane. ${ }^{56}$ We also reported the effects of three linker molecules, i.e., cysteamine, MPTMS and APTMS, on the attachment and growth of AuNPs on ITO surfaces. ${ }^{57}$ 

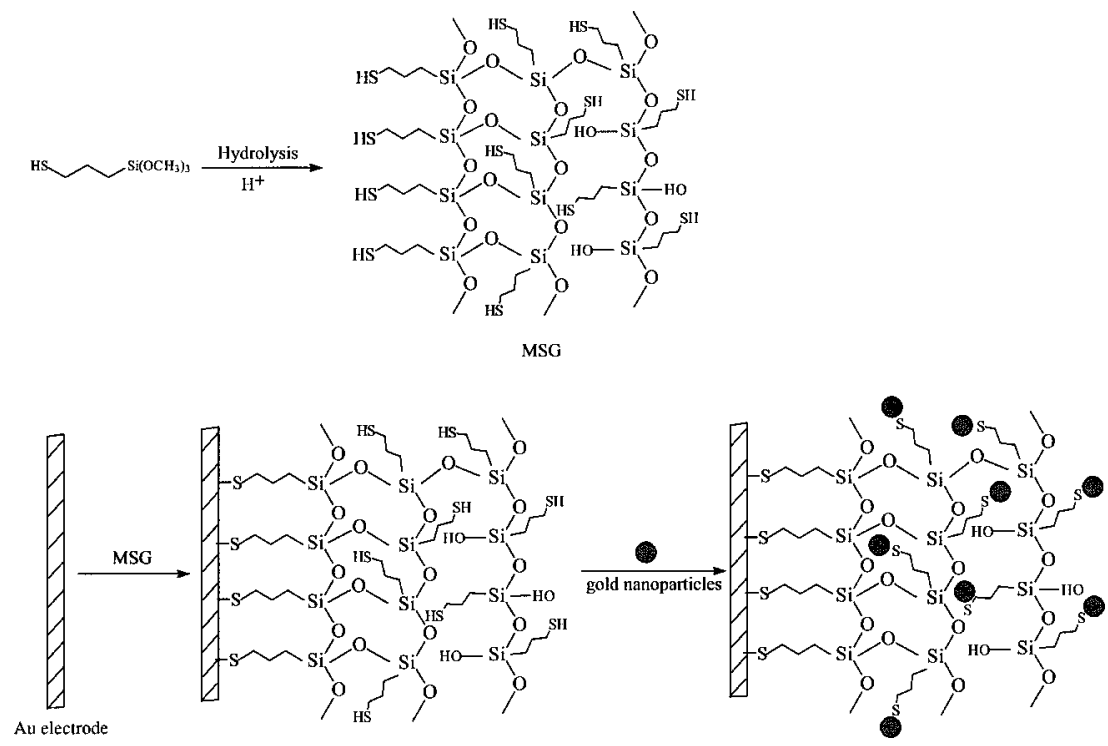

Fig. 2 Scheme of stepwise assembly of MPTMS sol-gel, AuNPs on a bare Au electrode. Reproduced from Ref. 58, copyright 2004, with permission from Elsevier.

\section{Self-assembly on a Three-dimensional Sol-gel Silica Network}

As a method to utilize the electrocatalytic ability of AuNPs, a sol-gel approach with MPTMS for modifying electrode surfaces is effective because the thiol tail groups in the 3D sol-gel silica network are able to immobilize AuNPs (Fig. 2). Wang and Wang reported that the direct electron transfer of cytochrome $c$ is possible with the thus-formed 20-nm AuNPs modified electrode. $^{58}$

Raj and coworkers expanded the applications of the sol-gel derived 3D silica network as a medium to modify AuNPs on $\mathrm{Au}$ electrodes. It has been demonstrated that the AuNPs self-assembled on the 3D sol-gel network to efficiently catalyze the oxidation of NADH in the absence of any mediators. ${ }^{59}$ In their actual preparation method, MPTMS sol prepared by dissolving MPTMS in a methanol/water, $0.1 \mathrm{M} \mathrm{HCl}$ mixture (1:3:3 molar ratio) was used to modify a gold electrode, and AuNPs of $2.6 \mathrm{~nm}$ diameter were self-assembled. ${ }^{59}$

The same preparation was used for the electrocaltalytic oxidation of glucose with the enlargement of AuNPs. ${ }^{60}$ For this purpose, a seed-mediated growth of AuNPs using hydroxylamine ${ }^{61}$ was performed after modifying the $3 \mathrm{D}$ sol-gel network with 2.6-nm Au nano-seeds. A thus-formed platform has permitted the enzyme-free amperometric sensing of glucose, ${ }^{60}$ ultrasensitive electrochemical sensing of hydrazine ${ }^{62}$ and the simultaneous electrochemical detection of ultratrace arsenic, mercury, and copper with the square-wave anodic stripping voltammetry. ${ }^{63}$ In addition, the morphology-dependent electrocatalytic activity of AuNPs was studied using ascorbate as a model compound. ${ }^{64}$

Furthermore, by integrating the dehydrogenase enzyme and AuNPs with the silica network, i.e., using the MPTMS sol-enzyme biocomposite to prepare the enzyme encapsulated sol-gel network followed by the self-assembly of AuNPs, a NADH-dependent dehydrogenase biosensor was developed for the amperometric biosensing of lactase and ethanol. ${ }^{65}$

Judging from the easier fabrication and controlling the size of AuNPs and successful applications, the sol-gel method would be promising for preparing 3D gold nanoelectrode ensembles. Jena and Raj also reported on the incorporation of $\mathrm{Au}$ nanoprisms, nanoperiwinkles ${ }^{66}$ and $\mathrm{Au}$ nanoflowers ${ }^{67}$ into the sol-gel network. Furthermore, they reported that platinum NPs can also be self-assembled onto the 3D sol-gel network, which was verified to be effective for the oxidation of methanol and the reduction of oxygen. ${ }^{68}$ Kalimuthu and John reported on the size-dependent electrocatalytic activity of AuNPs immobilized onto a 3D sol-gel network. ${ }^{69}$

\section{Polymer Materials for Modifying Metal NPs}

Some polymer materials can be used to incorporate or attach metal nanoparticles. Yu et al. reported nanostructured electrochemical sensors based on dense AuNP-films, which were prepared by infiltrating 4-(dimethylamino)pyridine-stabilized AuNPs into polyelectrolyte multilayers preassembled on ITO electrodes. ${ }^{70}$ By using poly(sodium 4-styrene sulfonate) and poly(allylamine hydrochloride) multilayers, dense AuNP-films were prepared and applied to the electrocatalytic oxidation of nitric oxide.

As another example of using polyelectrolyte, Halaoui's group has reported the nanostructured electrodes of polyacrylate-capped PtNPs in poly(diallyldimethylammonium)chloride on ITO or GC electrodes. ${ }^{71-73}$ Using the thus-formed electrodes, the absorption of atomic hydrogen, ${ }^{71}$ oxygen reduction $^{72}$ and the sensing of hydrogen peroxide at low surface density ${ }^{73}$ were studied.

Balamurugan and Chen reported that an $\mathrm{Ag}$ nanograins incorporated poly(3,4-ethlenedioxythiophene) (PEDOT) modified electrode could be prepared by a simple electrochemical method without using any stabilizers or reducing agent. ${ }^{74}$ Because the Ag nanograins incorporated PEDOT modified ITO electrode exhibited good electrocatalytic ability towards the reduction of hydrogen peroxide, it was applied to the amperometric detection of hydrogen peroxide. From the same group, Kumar et al. reported on the electrochemical synthesis of copper nanoparticles onto a poly(o-phenylenediamine)-coated GC electrode. ${ }^{75}$ Utilizing the electrocatalytic ability, the 
thus-fabricated electrode was applied to the ampermetric detection of hydrogen peroxide and nitrite.

In addition to the polyelectrolytes and conducting polymers, a functional mesoporous cross-linked polymer was used as a host for loading AuNPs, and the electrocatalytic behavior for the reduction of $\mathrm{H}_{2} \mathrm{O}_{2}$ was reported by Chandra et al. ${ }^{76}$ A spin-coat modified ITO with PEDOT-poly(styrene sulfonic acid) was used as a substrate to electrodeposit AuNPs; the electrode was applied to the electrocatalytic oxidation of NADH. ${ }^{77}$

\section{Integrated Nanoarchitectures with Metal NPs for Modifying Electrodes}

While several methods for dispersing metal NPs on electrodes two- or three-dimensionally are summarized above, further integrated nanoarchitectures have been developed. In particular, the use of carbon nanotubes (CNTs) has lead to interesting applications.

Dai et al. reported on electroanalysis using macro-, micro-, and nanochemical architectures on electrode surfaces. ${ }^{78}$ As the actual nanoarchitecture, AuNPs were deposited onto the surfaces of GC microspheres through electroless plating at first, and then the spheres were wired-up using multiwalled carbon nanotubes (MWCNTs) in a film on a macro GC electrode. With the fabricated electrode, macro-electrode votammetric behavior was obtained using $\sim 1 \%$ by mass of the expensive Au material that would be required for an equivalent Au film macrodisk electrode. In addition, it was applied to an analytical determination of the As(III) concentration.

The progress of metal NPs supported on CNTs was well-summarized in a review in 2006 by Wildgoose et al. ${ }^{79}$ As recent progress, $\mathrm{Qu}$ et al. reported the electrochemical fabrication of $\mathrm{Au}, \mathrm{Ag}, \mathrm{Pt}$ or Pd-NPs with a diameter of 40-60 nm using a MWCNTs/GC electrode, which was prepared by the ethanol cast method, followed by immersing into a solution of tetrakis(1-methyl-4-pyridyl)- $21 \mathrm{H}, 23 \mathrm{H}$ porphine, tetra-p-tosylate salt at first, then into a solution of noble metal complex (e.g., $\mathrm{AuCl}_{4}^{-}$), and finally electroreduction. ${ }^{80}$ The electrocatalytic activity of thus-formed PdNPs/MWCNTs/GC was investigated.

AgNPs in or on CNTs has been attracting some attention. AgNPs in MWCNT-modified ITO electrodes were prepared using Nafion, and applied to a surface-enhanced Raman scattering chemical sensor by Tsai et al. ${ }^{81}$ The same group also reported the coating of AgNPs in MWCNTs on ITO surfaces using alumina-coated silica for the same purpose. ${ }^{82}$ While electroreduction was used in these cases, ${ }^{81,82}$ it has been reported that, in the presence of carboxy group functionalized or poly(acrylic acid)-grafted CNTs, AgNPs were in situ generated from $\mathrm{AgNO}_{3}$ aqueous solutions without any additional reducing reagent. ${ }^{83}$ Thus-formed MWCNT/AgNP nanohybrids were cast-modified on a gold electrode, and the electrode was applied as a non-enzymatic hydrogen peroxide sensor by Zhao et al ${ }^{84}$

Chen et al. reported that a modification of the carboxy group functionalized CNTs (FCNTs) with PdNPs ${ }^{85}$ The PdNPs-FCNTs were fixed on a GC electrode with Nafion, and applied to a novel non-enzymatic electrogenerated chemiluminescence sensor for glucose. ${ }^{85}$ As another example of electrodeposition on CNTs, a composite of MWCNT-Nafion on GC electrodes could be used for the electrochemical deposition of PtNPs; Tsai and Hong reported preparation using a cyclic voltammetric method, and applied it to the oxidation of methanol. ${ }^{86}$

The layer-by-layer assembly can be used as a method to prepare integrated nanointerfaces. Using this technique,
AuNP/MWCNT thin film was fabricated and applied to the oxidation of methanol. ${ }^{87}$ Crespilho et al. reported a unique layer-by-layer approach for electroactive nanostructured membranes composed of polyamidoamine dendrimers, AuNPs, redox mediator, and poly(vinylsulfonic acid). ${ }^{88,89}$

The modification of metal NPs on micro carbon materials and fixation using Nafion on base electrodes would be a typical approach. However, the progress is not included in this review. A recent review by Baron et al. in 2009 summarized metal NPs deposited onto carbon microspheres, and their use for combinatorial electrochemistry and electroanalysis. ${ }^{90}$ In addition to carbon, colloid spheres of $\mathrm{TiO}_{2}{ }^{91,92}$ and polymer ${ }^{93}$ have been used for the assembly of PtNPs and raspberry-like hierarchical Au/Pt NPs.

As a unique approach, the synthesis of a NPs-integrated Au nanotubes array was reported by Zhou et al. ${ }^{94}$ By using a porous anodic alumina template with electroless deposition, the Au nanotube array was successfully prepared and applied to the electrochemical detection of glucose.

\section{Seed-mediated Growth Method for Modifying Electrode Surfaces}

\section{8-1 Development of a seed-mediated growth method for} surface modification

As overviewed above, various nanoarchitectures have been developed and performed to utilize metal NPs in functional modified electrodes for electroanalysis. Because capping reagents, linker molecules, and other binder materials (e.g., polymers) may affect the electron-transfer reactions more or less, successful results would be due to skillful nanodesigns to manifest the effective electrochemical responses with suppressing unfavorable effects. Therefore, as another approach, the attachment of metal NPs without using linker molecules or polymers should be effective. Thus, our group developed a seed-mediated growth method for surface modification with metal NPs particularly on ITO surfaces.

The seed-mediated growth method was originally developed by Murphy and coworkers for synthesizing $\mathrm{Au}$ nanorods in aqueous solution by the chemical reduction of $\mathrm{HAuCl}_{4} \cdot{ }^{95,96} \mathrm{We}$ applied this technique to the modification AuNPs on ITO surfaces. ${ }^{97}$ As the actual procedure, at first, a piece of ITO-coated glass was immersed into a seed solution prepared with $\mathrm{HAuCl}_{4}$, trisodium citrate and $\mathrm{NaBH}_{4}$, which contained Au nano-seed particles of $4 \mathrm{~nm} .{ }^{95}$ Next, it was immersed in the growth solution, which was prepared from $\mathrm{HAuCl}_{4}$, ascorbic acid, $\mathrm{NaOH}$, and cetyltrimethylammonium bromide (CTAB).

Figure $3 \mathrm{~A}$ shows the FE-SEM image observed after seeding, and Figs. $3 \mathrm{~B}$ and $3 \mathrm{C}$ show those observed after the seed-mediated growth treatment. In Figs. 3B and 3C, we can observe the grown AuNPs on ITO as white particles in the images on the background ITO crystals. Thus, it was clarified that AuNPs grew up to $c a .50-70 \mathrm{~nm}$ on the ITO surface from the Au nano-seed particles attached onto ITO (Fig. 3A) by immersion into the growth solution. ${ }^{97}$

The first attachment of nano-seed particles is expected to be promoted via physic-sorption of ultra-small NPs dispersed in the seed solution by simply dipping the substrates. ${ }^{98}$ The second process should involve crystal growth via the chemical reduction of $\mathrm{AuCl}_{4}^{-}$in the growth solution, which is inferred to gradually proceed around nano-seed particles on the substrates.

As shown in Fig. 3D, the FE-SEM image of AuNPs on the ITO surfaces pre-modified with MPTMS revealed significant differences in the morphology of the grown AuNPs. ${ }^{97}$ Because 

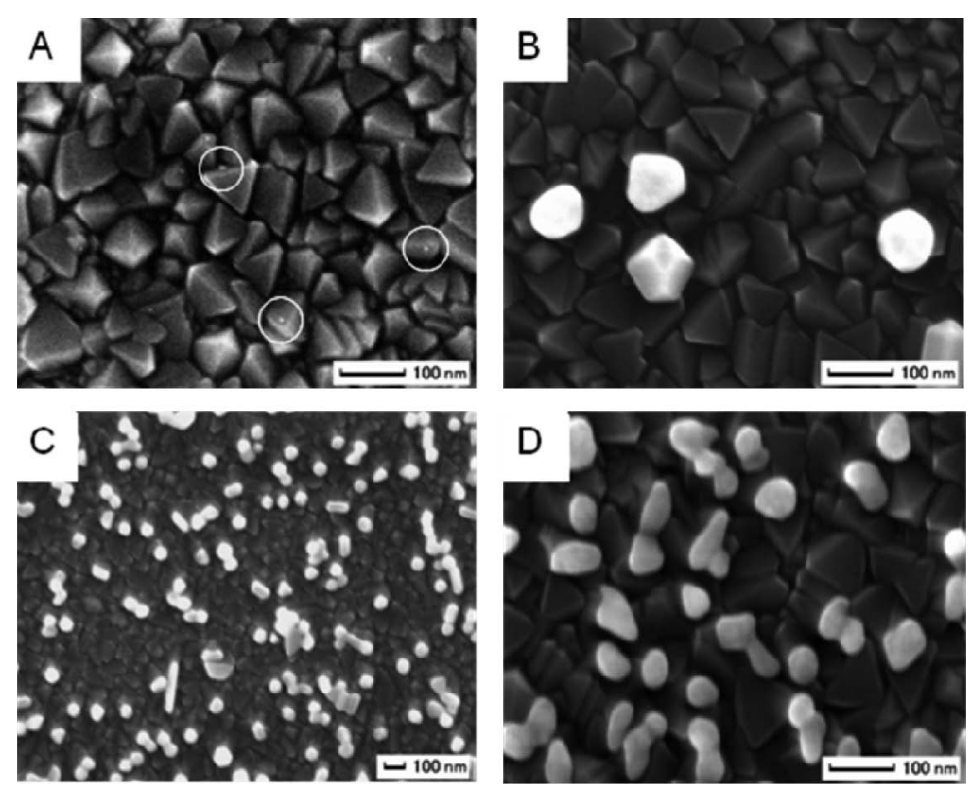

Fig. 3 FE-SEM images of the ITO surfaces. (A) After immersing ITO into the seed solution for $2 \mathrm{~h}$. The attachment of small seed particles is found, for example, in the white circles. (B) After immersing the ITO, which was treated in the seed solution, into the growth solution for $24 \mathrm{~h}$. (C) Zoom-out image of the ITO surface of (B). (D) ITO substrate modified at first with MPTMS, and then seed-mediated growth performed in the same manner as that for (B). Reproduced from Ref. 97, copyright 2005, with permission from the American Chemical Society.

the crystal-like structures of AuNPs were observed in the absence of MPTMS (Figs. 3B and 3C), the seed-mediated growth approach would be valid as a unique method for attaching Au nanocrystals onto the ITO surfaces without using linker molecules.

\subsection{Electrochemical applications of AuNP-attached ITO}

As inferred from the fact that the as-prepared AuNPs on the ITO surface (Figs. 3B and 3C) displayed a different morphology, the AuNP-attached ITO (AuNP/ITO) electrodes provided attractive electrochemical and electrocatalytic properties to promote heterogeneous electron-transfer kinetics. The quite low charge-transfer resistances of the AuNP/ITO electrodes were clarified from the electrochemical impedance results, and the typical charge-transfer resistance of the AuNP/ITO electrode was $c a .1 / 3$ of that of the AuNP/MPTMS/ITO electrode. ${ }^{99}$

The AuNP/ITO electrodes were used for observing the electrooxidation of uric acid, ascorbic acid, dopamine, norepinephrine and epinephrine. ${ }^{100}$ While the AuNP/MPTMS/ ITO electrodes depressed the selectivity in the determination of epinephrine in the presence of ascorbic acid due to the existence of the thiol monolayer, the AuNP/ITO electrodes could improve the detection sensitivity while retaining the selectivity.

The AuNP/ITO electrodes provided a bio-compatible matrix for the immobilization of hemoglobin $(\mathrm{Hb})$. Due to the promoted electron transfer of $\mathrm{Hb}$ by AuNPs, the Hb-immobilized AuNP/ITO electrodes exhibited an effective catalytic response to the reduction of $\mathrm{H}_{2} \mathrm{O}_{2}$ with good reproducibility and stability. ${ }^{101}$ Similarly, by immobilizing myoglobin (Mb), a mediator-free $\mathrm{H}_{2} \mathrm{O}_{2}$ sensor was developed. ${ }^{102}$

The effects of capping reagents on the electron-transfer reactions on the AuNP/ITO electrodes were investigated. ${ }^{103}$ In addition to the conventional preparation with the presence of CTAB in the growth solution, the presence of sodium dodecyl sulfate (SDS) was examined to find that the fabrication of
AuNP/ITO was also possible. As the results of systematic cyclic voltammetric measurements for the oxidation of anionic $\left[\mathrm{Fe}(\mathrm{CN})_{6}\right]^{4-}$ and the reduction of cationic $\left[\mathrm{Ru}\left(\mathrm{NH}_{3}\right)_{6}\right]^{3+}$, the electrochemical responses did not depend on the charges of the capping surfactants and the redox species. Thus, the capping of CTAB and SDS on AuNP/ITO was concluded to be quite weak. ${ }^{103}$ Utilizing the weak capping of CTAB on the grown AuNPs, the electrocatalytic activity of a 3D monolayer of 3-mercaptopropionic acid (MPA) assembled on AuNPs on the surface of ITO was studied. ${ }^{104}$ For the electrooxidation of ascorbic acid and dopamine, the 3D MPA monolayer showed obvious electrocatalytic promotion.

\subsection{Improved methods for the attachment and growth of AuNPs on ITO}

Some refined methods for improving or perturbing the seeding processes, i.e., the attachment of Au nano-seed particles, have been proposed because the seeding treatment is significant for nanostructuring grown AuNPs.

One approach is an in situ reduction method instead of seeding in the Au colloidal solution. It was demonstrated that, when the seeding procedure was modified to be carried out by an impregnation reduction of $\mathrm{AuCl}_{4}{ }^{-}$in the presence of the ITO substrate, the density of AuNPs directly grown on the surface was greatly improved. ${ }^{105}$ Figure 4 shows FE-SEM images of the AuNP-attached ITO surfaces prepared with the in situ reduction method. Following the initial attachment of the seed particles in Fig. 4A, we could see the growth of AuNPs with time on the nanostructured ITO surface (Figs. 4B and 4C). Repeating the growth treatment was also effective for increasing the size of the AuNPs (Fig. 4D). The thus-prepared AuNP/ITO electrodes exhibited high catalytic activity for the electrooxidation of nitric oxide. ${ }^{105}$

Another approach is an attempt to grow high-density AuNPs on ITO surfaces using a "touch" seeding technique instead of 

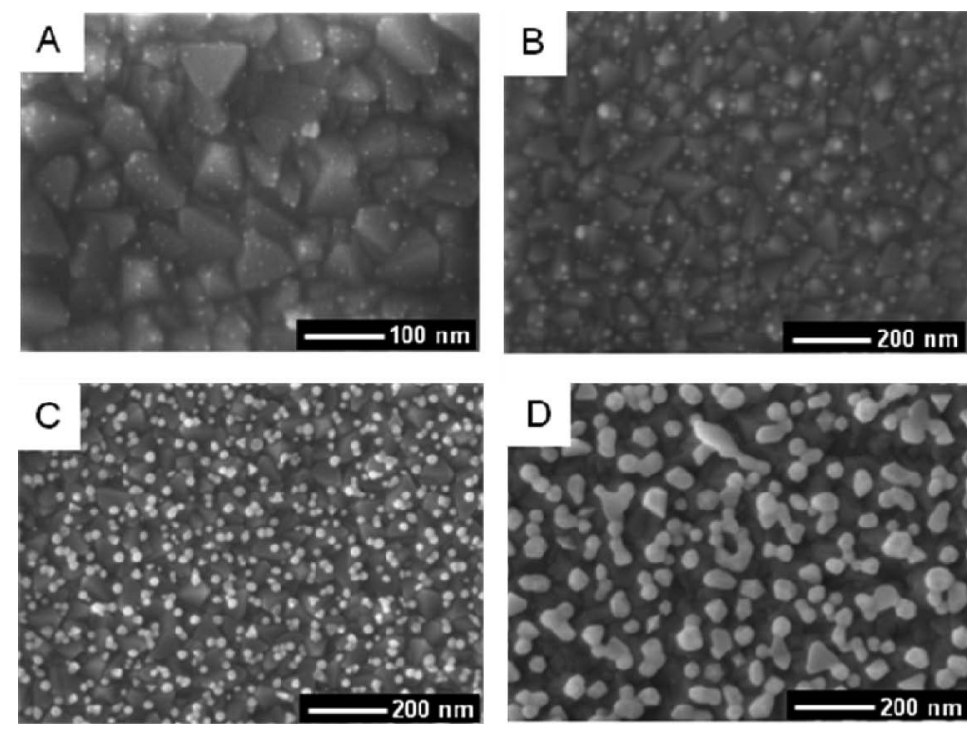

Fig. 4 FE-SEM images of AuNP-attached ITO surfaces. For seeding, the ITO substrate was immersed into a solution containing $\mathrm{AuCl}_{4}{ }^{-}$and citrate ions before reduction by $\mathrm{NaBH}_{4}$. (A) Observed just after seeding and (B, C) after the growth treatment for (B) $15 \mathrm{~min}$ and (C) $24 \mathrm{~h}$. (D) After repeating 24-h growth three times. Reproduced from Ref. 105, copyright 2005, with permission from Elsevier.
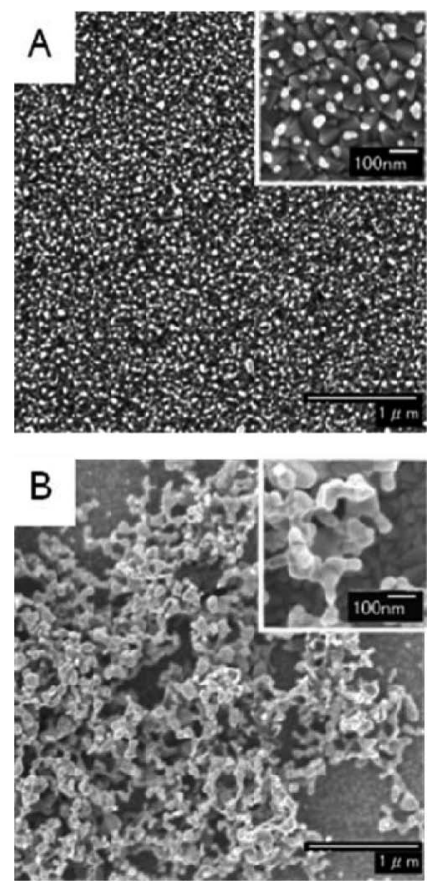

Fig. 5 FE-SEM images of AuNP-attached ITO surfaces prepared using the cast seed-mediated growth method. The repeated cycles of casting were (A) 3 and (B) 10 times. Reproduced from Ref. 108, copyright 2006, with permission from Elsevier.

the "normal" seeding in the seed-mediated growth procedure. ${ }^{106}$ Actually, surface that has already been covered with a drop of $\mathrm{Au}$ nano-seed solution was touched with tissue paper. In spite of the simplicity, this approach was found to be effective for the growth of high-density AuNPs, ${ }^{106}$ and was applied to observe the electrochemical responses of hydroquinone and p-aminophenol. ${ }^{107}$
The dense attachment of AuNPs could be also carried out using a cast seed-mediated growth method. ${ }^{108}$ Cast seeding with 3-cycles of dropping of the seed solution, followed by a treatment in the growth solution, was found to be suitable for preparing AuNP-attached ITO surfaces having a higher density and a narrower size distribution (Fig. 5A). On the other hand, a 10 -cycle cast seeding process formed the connected or networked nanostructures of AuNPs, as shown in Fig. 5B. ${ }^{108}$

Furthermore, adjusting the concentration of citrate ions in the seed solution from 1 to $50 \mathrm{mM}$ by adding trisodium citrate after preparation of the Au nano-seed solution, we could observe dramatic changes in the SEM images and actual colors of the ITO substrates. ${ }^{109}$

\subsection{Attachment and growth of Au nanoplates on ITO}

In the above section, the approaches and trials for preparation were mainly focused on the seeding process. However, a modification of the growth process should have a potential to alter the nanostructures of AuNPs on the ITO electrode surfaces.

As a new strategy for attaching Au nanoplates on ITO surfaces, we permitted the $2 \mathrm{D}$ crystal growth of $\mathrm{Au}$ through a reduction from Au nano-seed particles attached onto the ITO surface using poly(vinylpyrrolidone) (PVP) instead of CTAB as a capping reagent in the growth solution. ${ }^{110}$ By controlling the concentration of PVP, the formation of $\mathrm{Au}$ nanoplates was possible (Fig. 6A).

An improved method to attach Au nanoplates was recently reported, ${ }^{111}$ though the procedures have become a bit complicated, e.g., the growth solution contained both PVP and CTAB. The FE-SEM image prepared by the improved method is shown in Fig. 6B. As electrochemical applications of $\mathrm{Au}$ nanoplate-attached ITO electrode, the electrochemical responses were compared with those obtained with the AuNP/ITO electrode for the oxidation of dopamine and ascorbic acid. ${ }^{112}$ As a result, the higher electrocatalytic activity of $\mathrm{Au}$ nanoplate-attached ITO electrodes was confirmed. 

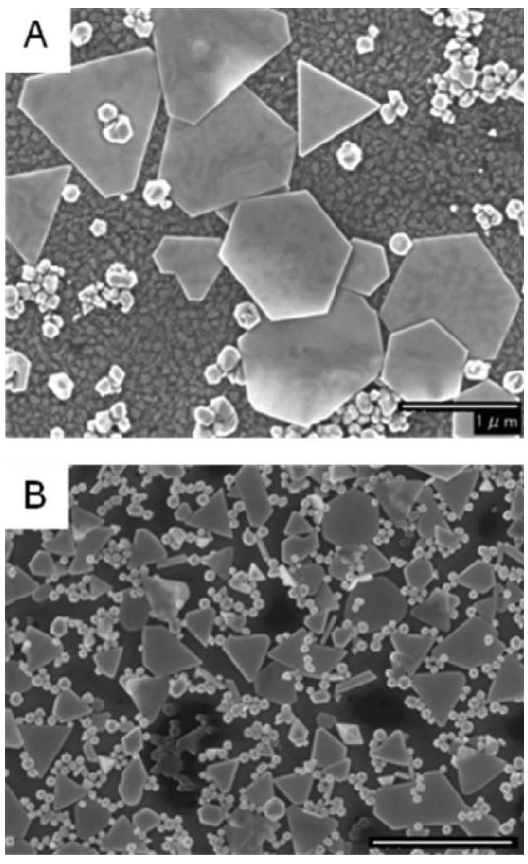

Fig. 6 FE-SEM images of Au nanoplates attached and grown on ITO surfaces prepared with (A) the original method ${ }^{110}$ and (B) the improved method. ${ }^{111}$ Scale bar is $1 \mu \mathrm{m}$. Reproduced from Refs. 110 and 111, copyright 2006 and 2009, with permission from the American Chemical Society.

\subsection{Attachment and growth of AuNPs on GC and $\mathrm{TiO}_{2}$}

We found that a modification of AuNPs was possible on all of the examined materials: GC, mica, stainless, epoxy resin, phenol resin, simple glass and $\mathrm{ZnO}$ film. FE-SEM observations of these materials revealed that the AuNPs attached and grew on the surfaces, though the grown size of the AuNPs and the formation of rod-like particles varied depending on the substrates. Thus, the attachment of the Au nano-seed particles by simple immersion into the Au colloid solutions is considered to proceed commonly via physic-sorption, not due to specific chemical bonding.

In the case of GC substrates, it was found that a dense attachment of AuNPs was possible with a shorter growth time compared with cases involving a modification of the ITO surfaces. ${ }^{113}$ The AuNP-attached GC electrodes prepared using the seed-mediated growth method were utilized for the electrochemical detection of nitrite. ${ }^{114}$

The seed-mediated growth method was utilized to attach AuNPs on mesoporous $\mathrm{TiO}_{2}$ films prepared using the liquid phase deposition process. ${ }^{115}$ While the $\mathrm{TiO}_{2}$ film showed a strong inhibition of the electron-transfer process of the $\left[\mathrm{Fe}(\mathrm{CN})_{6}\right]^{3-} /\left[\mathrm{Fe}(\mathrm{CN})_{6}\right]^{4-}$ redox couple, the attached AuNPs effectively improved the sluggish heterogeneous electron-transfer kinetics at the $\mathrm{TiO}_{2}$ film. ${ }^{115}$

\subsection{Attachment and growth of other metal NPs on ITO}

By applying the same seed-mediated growth method, Ag nanosphere and nanorod particles were successfully attached onto ITO surfaces. ${ }^{116}$ It was found that the formed nanostructures were very sensitive to the amount of ascorbic acid in the growth solution. When the concentration of ascorbic acid in the growth solution was $0.64 \mathrm{mM}$, AgNPs grew on the ITO surface while retaining moderate dispersion (Fig. 7A). In contrast, the formation of $\mathrm{Ag}$ nanorods and nanowires were observed to be
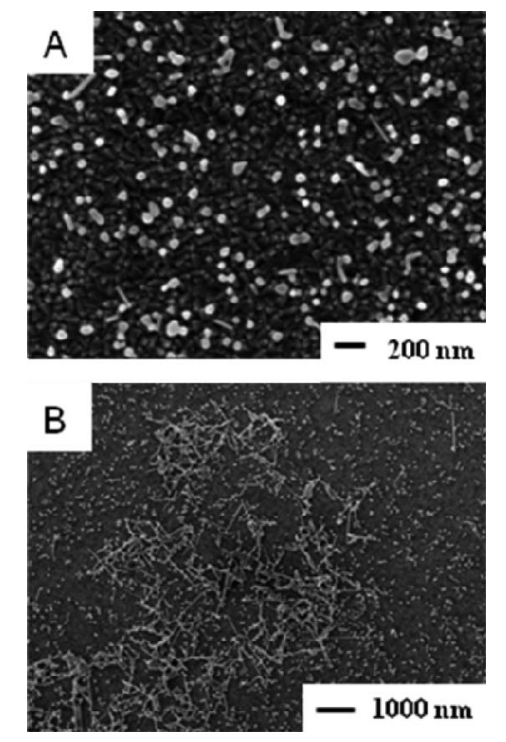

Fig. 7 FE-SEM images of AgNP-attached ITO surfaces. The ITO substrate was first immersed in the seed solution for $2 \mathrm{~h}$, and then into a growth solution containing (A) 0.64 or (B) $0.86 \mathrm{mM}$ of ascorbic acid for $24 \mathrm{~h}$. Reproduced from Ref. 116, copyright 2005, with permission from the American Chemical Society.

formed on the ITO surfaces (Fig. 7B) when the ascorbic acid was increased to $0.86 \mathrm{mM}$.

As for the AgNP/ITO electrodes, it was confirmed that the outer spheres of the $\mathrm{Ag}$ nanoparticles involved in the redox reaction showed the typical oxidation and reduction waves of $\mathrm{Ag}$ metal. ${ }^{116}$ In addition, the redox behavior of $\left[\mathrm{Fe}(\mathrm{CN})_{6}\right]^{3-} /\left[\mathrm{Fe}(\mathrm{CN})_{6}\right]^{4-}$ was improved on the AgNP/ITO electrode, reflecting the low electron-transfer resistance of $\mathrm{Ag}$. The AgNP/ITO electrode was also examined for the reduction of methyl viologen dication in order to discuss some native adsorption features of the fabricated AgNP/ITO electrodes. ${ }^{116}$

PdNPs were also successfully attached and grown on ITO surfaces using the seed-mediated growth method. ${ }^{117}$ Figure 8 shows FE-SEM images recorded after treating in the growth solution for $24 \mathrm{~h}$. The most characteristic feature of the formed nanostructure of Pd is that the Pd nanocrystals tend to stick to each other. In addition, aggregation occurred not only on the surface, but was also 3D, i.e., some nanocrystals were observed to grow above the basal nanocrystals. The nanostructure of Pd grown on ITO was quite different from the cases of $\mathrm{Au}$ and $\mathrm{Ag}$. Hence, it is inferred that the identity of the metal changed such aggregation characteristics in the growth process.

Due to dense attachment (Fig. 8B), the PdNP/ITO electrodes had significantly lowered charge-transfer resistances compared with that of a bare ITO. ${ }^{117}$ The electrocatalytic property of the PdNP/ITO electode was confirmed for the reduction of oxygen. In addition, some typical responses were observed in $0.5 \mathrm{M}$ $\mathrm{H}_{2} \mathrm{SO}_{4}$, reflecting both the characteristics of NPs and the thin layer on a nano-scale. ${ }^{117}$

The attachment of PtNPs on ITO was found to be possible by a rather simple method, i.e., a one-step in situ chemical reduction of $\mathrm{PtCl}_{4}{ }^{2-}$ by ascorbic acid without using CTAB, though the seed-mediated growth preparation failed. ${ }^{118}$ The attached PtNPs were spherical, showing an agglomerated nanostructure composed of small nanoclusters (Fig. 9). It was clarified that the PtNP/ITO electrodes had significant electrocatalytic properties for oxygen reduction and methanol oxidation. ${ }^{118}$ 

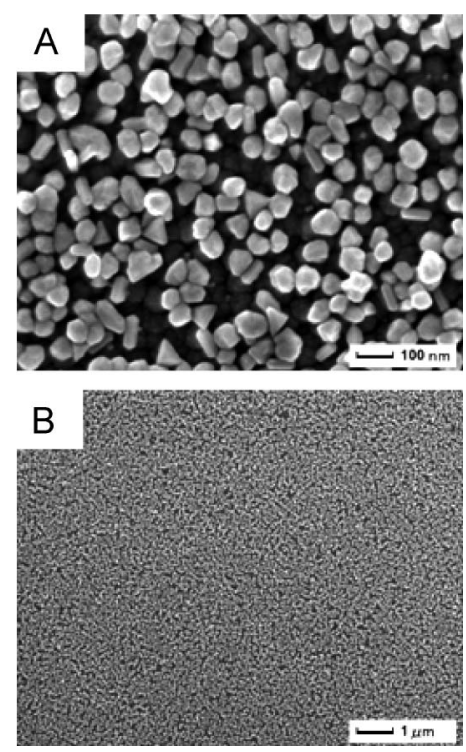

Fig. 8 FE-SEM images of PdNP-attached ITO surfaces. ITO substrates immersed into the seed solution for $2 \mathrm{~h}$ and then into the growth solution for $24 \mathrm{~h}$. (A) High-magnification and (B) lowmagnification images for the same surface. Reproduced from Ref. 117, copyright 2006, with permission from the American Chemical Society.
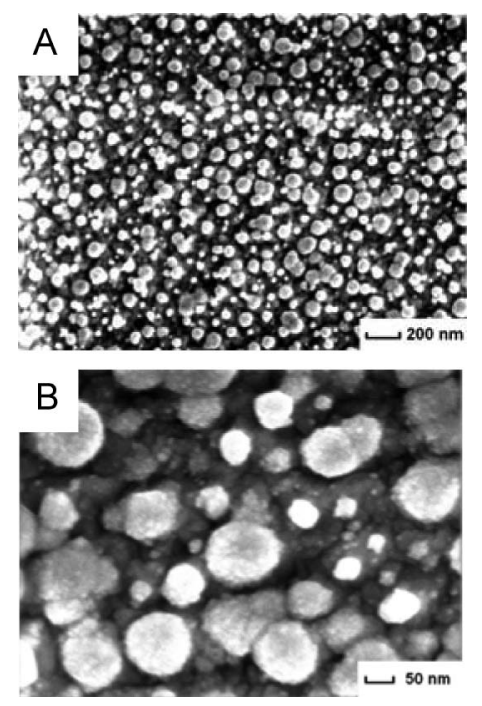

Fig. 9 FE-SEM images of PtNP-attached ITO surfaces. ITO substrate immersed in a growth solution containing $0.25 \mathrm{mM} \mathrm{K}_{2} \mathrm{PtCl}_{4}$ and $5 \mathrm{mM}$ ascorbic acid for $24 \mathrm{~h}$. (A) Low-magnification and (B) high-magnification images of the same surface. Reproduced from Ref. 118 , copyright 2006, with permission from the American Chemical Society.

The same in situ reduction method for preparing PtNPs was applied to the modification on GC electrodes. As a result, a thin continuous Pt film composed of small nanoclusters, which had a further agglomerated nanostructure of small grains, could be attached onto the GC surface (Fig. 10). ${ }^{119}$ The electrocatalytic ability of the Pt nanocluster film electrode for the methanol oxidation was apparently higher than those of the PtNP/ITO electrodes. ${ }^{119}$
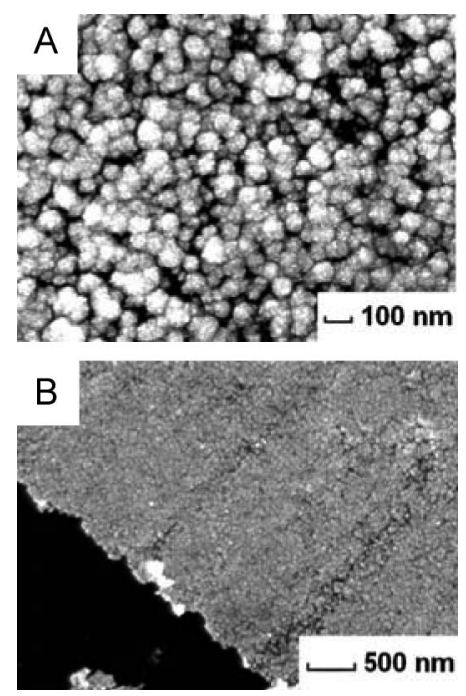

Fig. 10 FE-SEM images of Pt nanocluster film attached on GC. (A) High-magnification and (B) low-magnification images. The left lower corner of (B) was scratched to show the film thickness. Reproduced from Ref. 119, copyright 2007, with permission from Elsevier.

\subsection{Electrochemical measurements of biomolecules using} AuNP/ITO electrodes

In collaboration with Goyal's group in India, many successful results have been obtained using our AuNP/ITO electrodes and mainly with a differential pulse voltammetry technique for the electrochemical analysis of uric acid, ${ }^{120}$ paracetamol, ${ }^{121}$ atenolol, ${ }^{122}$ nandrolone, ${ }^{123}$ methylprednisolone acetate, ${ }^{124}$ salbutamol, ${ }^{125}$ and presnisolone. ${ }^{126}$ In addition, the simultaneous electrochemical determination of guanosine and guanosine$5^{\prime}$-triphosphate, ${ }^{127}$ adenosine and adenosine-5' ${ }^{\prime}$-triphosphate, ${ }^{128}$ dopamine and serotonin, ${ }^{129}$ and serotonin and 5-hydroxyindole ${ }^{130}$ were successfully carried out.

The AuNP/ITO electrodes might not be very tough, and the modified surfaces are difficult to renew if once damaged. However, Goyal's group used AuNP/ITO electrodes sent by post from Japan carefully, and obtained many results. This would be evidence of the practical robustness of AuNP/ITO electrodes prepared using our seed-mediated growth method.

\section{Concluding Remarks}

In this review, recent nanoarchitectures in metal NP-modified electrodes and their applications to electroanalysis have been summarized. The diversity of the fabrication methods may imply a complex aspect in the fixation and the utilization of metal NPs in functional modified electrodes for electroanalysis. However, compared with trials some years ago, the recent progress showed that fine nanodesigns have become possible to utilize the functions of metal NPs as electrode modifiers.

From recent progress in the electrochemical deposition of metal NPs, it seems that some fine tuning of the size, shape and density are now possible for some metal NPs and substrates. However, metal NPs prepared with the electrodeposition method tend to be larger than those that can be prepared with chemical methods; in addition, substrates have to be connected electrically during the preparations. Therefore, in spite of the size uniformity improved by the recent fine tuning of the conditions of electrodeposition, still the wet chemical synthesis would be 
significant for designing nanointerfaces.

As mentioned in the text, chemically prepared metal NPs can be regarded as nanocomposites, because they consist of gathered metal atoms and capping reagents. Also, some binder materials or additives are not, or less, conducting. Therefore, the designs of nanointerfaces for modified electrodes should be based on a delicate balance between fixation and electrical communication, particularly when we use smaller metal NPs prepared by chemical methods. This point would be important for refining the electrochemical observation and for making the best use of the functions of metal NPs.

To overcome the problems, in the final part of this review, we summarized some progress concerning our seed-mediated growth method as an interesting approach to attach metal NPs without using organic linker molecules or binder materials. Our trials have revealed that small metal nano-seed particles could be attached onto the surfaces of substrates (typically ITO and GC, but applicable to other materials) without using any binding reagents, and then nano-seed particles could be grown via chemical reductions while retaining a strong attachment. The simplicity of the preparation method, i.e., simply via two-step immersion into the seed solution first, and then in the growth solution, at ambient temperature, would be a significant merit concerning the proposed seed-mediated method for nanofabrication.

The lower charge-transfer resistances would be a key advantage in constructing electrode materials with metal NPs. In addition, the applicability to further surface modification of metal NPs should be beneficial for constructing functional interfaces. Therefore, nanocomposites consisting of metal NPs and base substrates prepared using the seed-mediated growth method would be regarded as being promising electrode materials.

\section{References}

1. D. Hernández-Santos, M. B. González-García, and A. Costa-García, Electroanalysis, 2002, 14, 1225.

2. E. Katz, I. Willner, and J. Wang, Electroanalysis, 2004, 16, 19.

3. C. M. Welch and R. G. Compton, Anal. Bioanal. Chem., 2006, 384, 601 .

4. N. S. Lawrence and H. Liang, "Nanostructured Materials in Electrochemistry", ed. A. Eftekhari, 2008, Chap. 12, Wiley-VCH, 435.

5. M. O. Finot, G. D. Braybrook, and M. T. McDermott, J. Electroanal. Chem., 1999, 466, 234.

6. M. S. El-Deab, T. Okajima, and T. Ohsaka, J. Electrochem. Soc., 2003, 150, A851.

7. M. S. El-Deab, T. Sotomura, and T. Ohsaka, J. Electrochem. Soc., 2005, 152, C1.

8. M. S. El-Deab, T. Sotomura, and T. Ohsaka, Electrochem. Commun., 2007, 9, 651.

9. X. Dai, O. Nekrassova, M. E. Hyde, and R. G. Compton, Anal. Chem., 2004, 76, 5924.

10. E. Majid, S. Hrapovic, Y Liu, K. B. Male, and J. H. T. Luong, Anal. Chem., 2006, 78, 762.

11. V. Carralero Sanz, M. Luz Mena, A. González-Cortés, P. Yáñez-Sedeño, and J. M. Pingarrón, Anal. Chim. Acta, $\mathbf{2 0 0 5}, 528,1$.

12. X. Dai and R. G. Compton, Anal. Sci., 2006, 22, 567.

13. L. Wang, W. Mao, D. Ni, J. Di, Y. Wu, and Y. Tu, Electrochem. Commun., 2008, 10, 673.

14. Y. Ma, J. Di, X. Yan, M. Zhao, Z. Lu, and Y. Tu, Biosens.
Bioelectron., 2009, 24, 1480.

15. J. Wang, L. Wang, J. Di, and Y. Tu, Talanta, 2009, 77, 1454.

16. Y. Zhao, Y. Wu, Y. Zhang, Z. Chen, X. Cao, J. Di, and J. Yang, Electroanalysis, 2009, 21, 939.

17. L. Rassaei, M. Sillanpää, R. W. French, R. G. Compton, and F. Marken, Electroanalysis, 2008, 20, 1286.

18. A. A. Isse, S. Gottardello, C. Maccato, and A. Gennaro, Electrochem. Commun., 2006, 8, 1707.

19. S. Thiagarajan, R. F. Yang, and S. M. Chen, Bioelectrochemistry, 2009, 75, 163.

20. S. B. Aoun and I. Taniguchi, Chem. Lett., 2008, 37, 936.

21. A. Safavi, N. Maleki, F. Tajabadi, and E. Farjami, Electrochem. Commun., 2007, 9, 1963.

22. A. Safavi, N. Maleki, F. Farjami, and E. Farjami, J. Electroanal. Chem., 2009, 626, 75.

23. S. Thiagarajan, B. W. Su, and S. M. Chen, Sens. Actuators, $B, \mathbf{2 0 0 9}, 136,464$.

24. Z. Wang, Q. Zhang, D. Kuehner, A. Ivaska, and L. Niu, Green Chem., 2008, 10, 907.

25. L. Lin, P. Qiu, X. Cao, and L. Jin, Electrochim. Acta, 2008, 53,5368 .

26. K. Murata, M. Suzuki, K. Kajiya, N. Nakamura, and H. Ohno, Electrochem. Commun., 2009, 11, 668.

27. R. G. Freeman, K. C. Grabar, K. J. Allison, R. M. Bright, J. A. Davis, A. P. Guthrie, M. B. Hommer, M. A. Jackson, P. C. Smith, D. G. Walter, and M. J. Natan, Science, 1995, 267, 1629.

28. G. Chumanov, K. Sokolov, B. W. Gregory, and T. M. Cotton, J. Phys. Chem., 1995, 99, 9466.

29. K. C. Grabar, R. G. Freeman, M. B. Hommer, and M. J. Natan, Anal. Chem., 1995, 67, 735.

30. W. Cheng, S. Dong, and E. Wang, Anal. Chem., 2002, 74, 3599.

31. W. Cheng, S. Dong, and E. Wang, Langmuir, 2002, 18, 9947.

32. K. H. Lee, K. M. Huang, W. L. Tseng, T. C. Chiu, Y. W. Lin, and H. T. Chang, Langmuir, 2007, 23, 1435.

33. S. Melzer, R. Resch, B. E. Koel, M. E. Thompson, A. Madhukar, A. A. G. Requicha, and P. Will, Langumuir, 2001, 17, 1713.

34. Y. Jin, X. Kang, Y. Song, B. Zhang, G. Cheng, and S. Dong, Anal. Chem., 2001, 73, 2843.

35. L. Wang and E. Wang, Electrochem. Commun., 2004, 6, 25.

36. M. Brust, M. Walker, D. Bethell, D. J. Schiffrin, and R. Whyman, J. Chem. Soc., Chem. Commun., 1994, 802.

37. A. C. Templeton, W. P. Wuelfing, and R. W. Murray, Acc. Chem. Res., 2000, 33, 27.

38. M. Tominaga, T. Shimazoe, M. Nagashima, and I. Taniguchi, Chem. Lett., 2005, 34, 202.

39. M. Tominaga, T. Shimazoe, M. Nagashima, and I. Taniguchi, Electrochem. Commun., 2005, 7, 189.

40. M. Tominaga, T. Shimazoe, M. Nagashima, and I. Taniguchi, J. Electroanal. Chem., 2008, 615, 51.

41. Y. Yamamoto, H. Shiigi, and T. Nagaoka, Electroanalysis, 2005, 17, 2224.

42. W. Chen, J. Kim, S. Sun, and S. Chen, Chem. Phys. Phys. Chem., 2006, 8, 2779.

43. W. Chen, J. Kim, S. Sun, and S. Chen, Langmuir, 2007, 23 , 11303.

44. J. A. Harnisch, A. D. Pris, and M. D. Porter, J. Am. Chem. Soc., 2001, 123, 5829.

45. L. Zhang, X. Jiang, E. Wang, and S. Dong, Biosens. Bioelectron., 2005, 21, 337.

46. L. Zhang and X. Jiang, J. Electroanal. Chem., 2005, 583, 292. 
47. G. Hu, D. Zhang, W. Wu, and Z. Yang, Colloids Surf., B, 2008, 62, 199.

48. C. R. Raj, T. Okajima, and T. Ohsaka, J. Electroanal. Chem., 2003, 543, 127.

49. G. Hu, Y. Ma, Y. Guo, and S. Shao, Electrochim. Acta, 2008, 53, 6610.

50. G. Wang, W. Wang, J. Wu, H. Liu, S. Jiao, and B. Fang, Microchim. Acta, 2009, 164, 149.

51. A. Sivanesan, P. Kannan, and S. A. John, Electrochim. Acta, 2007, 52, 8118.

52. P. Kannan and S. A. John, Anal. Biochem., 2009, 65, 386.

53. L. Wang, J. Bai, P. Huang, H. Wang, L. Zhang, and Y. Zhao, Electrochem. Commun., 2006, 8, 1825.

54. L. Wang, J. Bai, P. Huang, H. Wang, L. Zhang, and Y. Zhao, Electrochem. Commun., 2006, 8, 1035.

55. M. S. El-Deab, T. Okajima, and T. Ohsaka, J. Electrochem. Soc., 2006, 153, E201.

56. B. Ballarin, M. C. Cassani, E. Scavetta, and D. Tonelli, Electrochim. Acta, 2008, 53, 8034.

57. M. Oyama, A. Orimo, and K. Nouneh, Electrochim. Acta, 2009, 54, 5024.

58. L. Wang and E. Wang, Electrochem. Commun., 2004, 6, 49.

59. C. R. Raj and B. K. Jena, Chem. Commun., 2005, 2005.

60. B. K. Jena and C. R. Raj, Chem. Eur. J., 2006, 12, 2702.

61. K. R. Brown and M. J. Natan, Langmuir, 1998, 14, 726.

62. B. K. Jena and C. R. Raj, J. Phys. Chem. C, 2007, 111, 6228.

63. B. K. Jena and C. R. Raj, Anal. Chem., 2008, 80, 4836.

64. B. K. Jena and C. R. Raj, Electrochem. Commun., 2008, 10, 951.

65. B. K. Jena and C. R. Raj, Anal. Chem., 2006, 78, 6332.

66. B. K. Jena and C. R. Raj, J. Phys. Chem. C, 2007, 111, 15146.

67. B. K. Jena and C. R. Raj, Chem. Mater., 2008, 20, 3546.

68. B. K. Jena and C. R. Raj, J. Phys. Chem. C, 2008, 112, 3496.

69. P. Kalimuthu and S. A. John, J. Electroanal. Chem., 2008, 617, 164

70. A. Yu, Z. Liang, J. Cho, and F. Caruso, Nano Lett., 2003, 3, 1203.

71. M. Z. Markarian, M. E. Harakeh, and L. I. Halaoui, J. Phys. Chem. B, 2005, 109, 11616.

72. Z. G. Estephan, L. Alawieh, and L. I. Halaoui, J. Phys. Chem. C, 2007, 111, 8060

73. P. Karam and L. I. Halaoui, Anal. Chem., 2008, 80, 5441.

74. A. Balamurugan and S. M. Chen, Electroanalysis, 2009, $21,1419$.

75. S. A. Kumar, P. H. Lo, and S. M. Chen, J. Electrochem. Soc., 2009, 156, E118.

76. D. Chandra, B. K. Jena, C. R. Raj, and A. Bhaumik, Chem. Mater., 2007, 19, 6290.

77. K. M. Manesh, P. Santhosh, A. Gopalan, and K. P. Lee, Talanta, 2008, 75, 1307.

78. X. Dai, G. G. Wildgoose, C. Salter, A. Crossley, and R. G. Compton, Anal. Chem., 2006, 78, 6102.

79. G. G. Wildgoose, C. E. Banks, and R. G. Compton, Small, 2006, 2, 182

80. J. Qu, H. Chen, and S. Dong, Electroanalysis, 2008, 20, 2410.

81. Y. C. Tsai, P. C. Hsu, Y. W. Lin, and T. M. Wu, Sens. Actuators, B, 2009, 138, 5 .

82. Y. C. Tsai, P. C. Hsu, Y. W. Lin, and T. M. Wu, Electrochem. Commun., 2009, 11, 542.

83. C. Gao, W. Li, Y. Z. Jin, and H. Kong, Nanotechnology, 2006, 17, 2882.
84. W. Zhao, H. Wang, X. Qin, X. Wang, Z. Zhao, Z. Miao, L. Chen, M. Shan, Y. Fang, and Q. Chen, Talanta, 2009, 80, 1029.

85. X. Chen, Z. Cai, Z. Lin, T. Jia, H. Liu, Y. Jiang, and X Chen, Biosens. Bioelectron., 2009, 24, 3475.

86. Y. C. Tsai and Y. H. Hong, J. Solid State Electrochem., 2008, 12, 1293.

87. J. Kim, S. W. Lee, P. T. Hammong, and Y. Shao-Horn, Chem. Mater., 2009, 21, 2993.

88. F. N. Crespilho, M. E. Ghica, M. Florescu, F. C. Nart, O. N. Oliveira Jr., and C. M. A. Brett, Electrochem. Commun. 2006, 8, 1665.

89. F. N. Crespilho, M. E. Ghica, V. Zucolotto, F. C. Nart, O. N. Oliveira Jr., and C. M. A. Brett, Electroanalysis, 2007, 19,805 .

90. R. Baron, G. G. Wildgoose, and R. G. Compton, J. Nanosci. Nanotechnol., 2009, 9, 2274

91. S. Guo, S. Dong, and E. Wang, J. Phys. Chem. C, 2009, $113,5485$.

92. D. Wen, S. Guo, J. Zhai, L. Deng, W. Ren, and S. Dong, $J$ Phys. Chem. C, 2009, 113, 13023.

93. L. Zhang, L. Wang, S. Guo, J. Zhai, S. Dong, and E. Wang, Electrochem. Commun., 2009, 11, 258.

94. Y. Zhou, S. Yang, Q. Qian, and X. Xia, Electrochem Commun., 2009, 11, 216.

95. N. R. Jana, L. Gerheart, and C. J. Murphy, J. Phys. Chem. $B, \mathbf{2 0 0 1}, 105,4065$.

96. B. D. Busbee, S. Obare, and C. J. Murphy, Adv. Mater., 2003, 15, 414

97. M. Kambayashi, J. Zhang, and M. Oyama, Cryst. Growth Des., 2005, 5, 81 .

98. H. Bönnemann, G. Braun, W. Brijoux, R. Brinkmann, A Schulze Tilling, K. Seevogel, and K. Siepen, J. Organomet. Chem., 1996, 520, 143.

99. J. Zhang, M. Kambayashi, and M. Oyama, Electrochem. Commun., 2004, 6, 683 .

100.J. Zhang, M. Kambayashi, and M. Oyama, Electroanalysis, 2005, 17, 408 .

101.J. Zhang and M. Oyama, Electrochem. Acta, 2004, 50, 85.

102.J. Zhang and M. Oyama, J. Electroanal. Chem., 2005, 577, 273.

103. T. Horibe, J. Zhang, and M. Oyama, Electroanalysis, 2007 , $19,847$.

104.J. Zhang and M. Oyama, Electrochem. Commun., 2007, 9, 459.

105.J. Zhang and M. Oyama, Anal. Chim. Acta, 2005, 540, 299.

106. A. Ali Umar and M. Oyama, Cryst. Growth Des., 2005, 5, 599.

107. A. Ali Umar and M. Oyama, Indian J. Chem., Sect. A, 2005, 44, 938 .

108. A. Ali Umar and M. Oyama, Appl. Surf. Sci., 2006, 253, 2196.

109. A. Ali Umar and M. Oyama, Appl. Surf. Sci., 2006, 253, 2933.

110. A. Ali Umar and M. Oyama, Cryst. Growth Des., 2006, 6, 818.

111. A. Ali Umar, M. Oyama, M. Mat Salleh, and B. Yeop Majlis, Cryst. Growth Des., 2009, 9, 2835.

112. R. N. Goyal, A. Ali Umar, and M. Oyama, J. Electroanal. Chem., 2009, 631, 58.

113.M. Oyama, S. Yamaguchi, and J. Zhang, Anal. Sci., 2009, $25,249$.

114. Y. Cui, C. Yang, W. Zeng, M. Oyama, W. Pu, and J. Zhang, Anal. Sci., 2007, 23, 1421.

115.J. Zhang and M. Oyama, Electrochem. Solid-State Lett., 
$\mathbf{2 0 0 5}, 8, \mathrm{E} 49$.

116.G. Chang, J. Zhang, M. Oyama, and K. Hirao, J. Phys. Chem. B, 2005, 109, 1204.

117.G. Chang, M. Oyama, and K. Hirao, J. Phys. Chem. B, 2006, 110, 20362.

118. G. Chang, M. Oyama, and K. Hirao, J. Phys. Chem. B, 2006, 110, 1860.

119. G. Chang, M. Oyama, and K. Hirao, Thin Solid Films, 2007, 515, 3311.

120. R. N. Goyal, M. Oyama, A. Sangal, and S. P. Singh, Indian J. Chem., Sect. A, 2005, 44, 945.

121.R. N. Goyal, V. K. Gupta, M. Oyama, and N. Bachheti, Electrochem. Commun., 2005, 7, 803.

122. R. N. Goyal, V. K. Gupta, M. Oyama, and N. Bachheti, Electrochem. Commun., 2006, 8, 65.

123. R. N. Goyal, M. Oyama, A. Tyagi, and S. P. Singh, Talanta,
2007, 72, 140.

124.R. N. Goyal, M. Oyama, A. Ali Umar, A. Tyagi, and N. Bachheti, J. Pharm. Biomed. Anal., 2007, 44, 1147.

125. R. N. Goyal, M. Oyama, and S. P. Singh, J. Electroanal. Chem., 2007, 611, 140.

126. R. N. Goyal, M. Oyama, N. Bachheti, and S. P. Singh, Bioelectrochemistry, 2009, 74, 272.

127.R. N. Goyal, M. Oyama, and A. Tyagi, Anal. Chim. Acta, 2007, 581, 32 .

128. R. N. Goyal, M. Oyama, and S. P. Singh, Electroanalysis, 2007, 19, 575.

129.R. N. Goyal, V. K. Gupta, M. Oyama, and N. Bachheti, Talanta, 2007, 72, 976.

130. R. N. Goyal, M. Oyama, V. K. Gupta, S. P. Singh, and R. A. Sharma, Sens. Actuators, 2008, 134, 816. 